\title{
Cost-efficient observation of working postures from video recordings - more videos, more observers or more views per observer?
}

\author{
Svend Erik Mathiassen ${ }^{\mathrm{a}^{*}}$, Per Liv $^{\mathrm{a}}$ and Jens Wahlström ${ }^{\mathrm{ab}}$ \\ ${ }^{a}$ Centre for Musculoskeletal Research, Department of Occupational and Public Health Sciences, University of \\ Gävle, SE-80176 Gävle, Sweden \\ ${ }^{\mathrm{b}}$ Department of Public Health \& Clinical Medicine, Occupational and Environmental Medicine, Umeå \\ University, SE-90185 Umeå, Sweden
}

\begin{abstract}
In ergonomics, assessing the working postures of an individual by observation is a very common practice. The present study investigated whether monetary resources devoted to an observational study should preferably be invested in collecting many video recordings of the work, or in having several observers estimate postures from available videos multiple times. On the basis of a data set of observed working postures among hairdressers, necessary information in terms of posture variability, observer variability, and costs for recording and observing videos was entered into equations providing the total cost of data collection and the precision (informative value) of the resulting estimates of two variables: percentages time with the arm elevated $<15$ degrees and $>90$ degrees. In all 160 data collection strategies, differing with respect to the number of video recordings and the number of repeated observations of each recording, were simulated and compared for cost and precision. For both posture variables, the most cost-efficient strategy for a given budget was to engage 4 observers to look at available video recordings, rather than to have one observer look at more recordings. Since the latter strategy is the more common in ergonomics practice, we recommend reconsidering standard practice in observational posture assessment.
\end{abstract}

Keywords: resource consumption, mean exposure, risk assessment, posture observation, efficiency

\section{Introduction}

In ergonomics research and practice, working postures are often assessed by observations, either in the field or from video recordings of work [4, 10, 20]. While modern technology may, in some years, offer cheap and user-friendly devices for direct recordings of working postures, observations can be expected to survive as a frequently used tool, at least among practitioners in working life.

Often, the intended outcome of the posture assessment is an estimate of the mean value of some posture variable believed to be relevant to the specific purpose of the investigation, typically assessment of risk for contracting musculoskeletal disorders. As with any collection of occupational exposure data, posture assessments face resource constraints, usually in terms of a limited budget. This presents the investigator with the challenge of using available resources efficiently, i.e. obtaining as much information as possible about the desired posture variable(s) at the allotted cost.

In this context, information can be expressed by the reciprocal standard deviation (SD) of the obtained mean posture estimate, reflecting that posture estimates with a low precision (a large SD) does not contain as much information as posture estimates with a better precision (a small SD). For observations, this standard deviation (or variance) depends both on the "biological" posture variability within and between workers due to differences in work tasks and work technique, and on the methodological variability introduced by differences in posture ratings within and between observers [4].

* Corresponding author. Email: smn@hig.se 
Provided that the contribution of each of these sources of variability is known, in terms of a socalled variance component, the precision of the mean can be assessed, both retrospectively for data collections already done, and prospectively for studies in the planning phase $[9,18]$. Several studies have been devoted to identifying principles for statistically efficient design of an exposure data collection strategy on the basis of variance components (e.g. [3, 5, 8, 13, 14, 19, 22]), but few have specifically addressed working postures (e.g. $[2,6,7,11,15])$, and then only on the basis of direct technical recordings using inclinometers. Thus, the influence of observer variability on the overall precision of an observed posture mean value is largely unknown, even if a few studies report variability data to feed a discussion of this issue $[1$, 9, 16]. Unfortunately, a majority of studies investigating observer variability report their findings in terms of metrics, typically kappa coefficients, that are not useful for assessing statistical precision or contemplating alternative data collection strategies $[4,20]$, even if they consistently show that both within- and between-observer disagreement is a serious concern for most posture variables.

Furthermore, different stages in a data collection process may entail different costs, and so the most efficient strategy in a statistical sense may not necessarily be the most cost-efficient [12]. Very little research has been devoted to investigating trade-offs between cost and statistical efficiency in data collection [17], and no studies have so far focused on the generic issue in video-based observation of whether resources - as constrained by a limited budget - should be allocated to collecting "many" video recordings and have them observed by "few" observers, or to a more meticulous observation of fewer video recordings.

The present study examined the cost-efficiency of alternative allocations of resources between video recordings, observers and repeated observations by each observer. The study took on the common case in ergonomics practice of estimating the mean posture (in casu arm elevation) of a specific individual, for instance in order to determine whether that individual complies with an exposure threshold limit or whether the individual has benefited from an ergonomics intervention.

\section{Methods}

\subsection{Materials}

Upper arm elevation was measured using inclinometers for four full work shifts in each of 28 female hairdressers, and expressed through a number of posture and velocity variables [23]. On two randomly selected days, work was also recorded continuously on video for a randomly selected half hour during the day. The video recordings from five randomly selected hairdressers were picked out for observation. Four observers, trained independently of one another, observed each of these 10 half-hour videos using a work sampling approach. In total 120 frames per half-hour video, interspersed by a fixed $15 \mathrm{~s}$ interval, were presented to the observer on a computer screen, and she was required to estimate and type the elevation angle of the upper right arm. This particular work sampling approach was shown to have a good cost-efficiency compared to a number of other observation approaches [18]. On the basis of the 120 posture estimates, percentages of time with the arm elevated $<15$ degrees ("\%time $<15$ ") and $>90$ degrees ("\%time $>90 ")$ were determined. All four observers assessed all 10 video recordings twice on different days.

\subsection{Precision}

For each of the two posture variables, variances between and within observers were estimated on the basis of the available 80 half-hour estimates (5 hairdressers x 2 days per hairdresser $\mathrm{x} 4$ observers $\mathrm{x}$ 2 views per observer), using REML algorithms in the model shown by Eq. (1):

$\mathrm{y}_{\mathrm{ijkl}}=\mu+\alpha_{\mathrm{i}}+\beta_{\mathrm{j}(\mathrm{i})}+\gamma_{\mathrm{k}}+\varepsilon_{\mathrm{l}(\mathrm{ijk})}$

where $\mu$ is the overall mean value, $\alpha_{i}$ is the effect of the $\mathrm{i}$ :th hairdresser, $\beta_{\mathrm{j}(\mathrm{i})}$ is the effect of the $\mathrm{j}$ :th video recording within the $i$ :th hairdresser, $\gamma_{\mathrm{k}}$ is the effect of the k:th observer, and the error term $\varepsilon_{1(\mathrm{ijk})}$ represents the effect of the 1:th replicate of a particular observation. The corresponding variance components $\sigma_{\text {bs }}^{2}, \sigma_{\text {ws }}^{2}, \sigma_{\text {bo }}^{2}, \sigma_{\text {wo }}^{2}$ are the variances between subjects (hairdressers), within subjects, between observers and within observers, respectively. While the model shown in Eq. (1) extracted both "biological" and "methodological" variance components in the observed data set, the "true" posture means and biological variance 
components between and within hairdressers, i.e. $\sigma_{b s}^{2}$ and $\sigma_{\text {ws }}^{2}$, were instead obtained from the inclinometer recordings [23]. Thus, from the observation data set only the observer variabilities, $\sigma_{\text {bo }}^{2}$ and $\sigma_{\text {wo }}^{2}$, were used for further analysis. On the basis of the variance components, the variance of a posture mean value for one observed individual, $\sigma_{\mu}^{2}$, can be estimated as:

$\sigma_{\mu}^{2}=\sigma_{w s}^{2} / n_{d}+\sigma_{b o}^{2} / n_{o}+\sigma^{2}{ }_{w o} /\left(n_{d} \cdot n_{o} \cdot n_{r}\right)$

where $n_{d}, n_{o}$, and $n_{r}$ is the number of collected video recordings, observers, and views per observer, respectively. The standard deviation of the mean, $\mathrm{SD}_{\mu}$, is the square root of this variance, and precision in this context was assessed as $1 / \mathrm{SD}_{\mu}$.

\subsection{Cost}

The total cost, $\mathrm{C}_{\mathrm{T}}$, for data collection was assessed as the sum of labour costs for filming (proportional to the number of half-hour video recordings) and labour costs for observation (proportional to the total number of viewed recordings) according to the following cost model:

$\mathrm{C}_{\mathrm{T}}=\mathrm{C}_{\mathrm{F}} \cdot \mathrm{n}_{\mathrm{d}}+\mathrm{C}_{\mathrm{O}} \cdot \mathrm{n}_{\mathrm{d}} \cdot \mathrm{n}_{\mathrm{o}} \cdot \mathrm{n}_{\mathrm{r}}$

where $C_{F}$ and $C_{O}$ are the unit costs for obtaining one video recording and for observing one recording once, respectively. These unit labour costs were calculated on basis of average reported times spent filming or observing one video, multiplied by the hourly salary of the staff.

Total cost, $\mathrm{C}_{\mathrm{T}}$, according to Eq. (3), and precision, on the basis of Eq. (2), was then estimated for all 160 combinations of 1-10 video recordings observed by 1-4 observers, each repeating their observations 1-4 times.

\section{Results}

For the variable $\%$ time $<15$, the overall mean, $\mu$, was $24 \%$ time, and variances between days (within hairdresser), between observers and within observer, i.e. $\sigma_{\text {ws }}^{2}, \sigma_{\text {bo }}^{2}$ and $\sigma_{\text {wo }}^{2}$, were $73 \%$ time ${ }^{2}, 87 \%$ time ${ }^{2}$ and $131 \%$ time $^{2}$, respectively. The corresponding mean and variance components for \%time $>90$ were $2 \%$ time, $3 \%$ time $^{2}, 0 \%$ time $^{2}$ and $9 \%$ time $^{2}$. The unit cost, $\mathrm{C}_{\mathrm{F}}$, for collecting one half-hour video was $36 €$, and that for observing it, $\mathrm{C}_{\mathrm{O}}, 7 €$.
For $\%$ time $<15$, inspection of the 160 sets of total cost and corresponding statistical precision showed that for all budgets up to $600 €$, the most costefficient strategy was to let 4 observers look at the collected video recordings one time each. At total budgets of $200 €, 400 €$ and $600 €$ this observation strategy allowed for 3, 6 and 9 video recordings, respectively. Investing $200 €, 400 €$ and $600 €$ in collecting instead as many video recordings as possible (4, 9 and 13, respectively) and have each of them observed only once by one observer, decreased the precision (i.e. $1 / \mathrm{SD}_{\mu}$ ) of the resulting mean exposure estimate to only $64 \%, 60 \%$ and $57 \%$, respectively, of that obtained using the 4-observer strategy. The one-observer one-view strategy was the least cost-efficient of all investigated alternatives, irrespective of the total allowed budget. Increasing the budget from $200 €$ to $600 €$ increased precision when using the most cost-efficient data collection strategy - from $0.13 \%$ time $^{-1}$ to $0.17 \%$ time $^{-1}$, i.e. by $30 \%$.

For \%time $>90$, the optimal strategy among those investigated was also to let 4 observers look at the collected video recordings one time each, shared by the equally cost-efficient alternatives of 2 observers repeating their observations twice, or having one observer observing 4 times. The affordable number of video recordings at $200 €, 400 €$ and $600 €$ budgets were obviously the same as those reported above for $\%$ time $<15$. However, for $\%$ time $>90$ the decreases in precision from selecting more recordings but only one observation by one observer were less severe: down to $78 \%, 83 \%$ and $81 \%$ of the optimal value. Increasing the budget from $200 €$ to $600 €$ in this case improved precision by $72 \%$, from $0.73 \%$ time $^{-1}$ to $1.26 \%$ time $^{-1}$.

\section{Discussion}

Our results clearly show that when only a limited budget is available for observation-based assessment of postures, it can be more cost-efficient to invest resources in a more meticulous observation procedure than to spend money in recording more videos. This is, to our best knowledge, not realized by researchers and practitioners, considering the large number of observation studies relying on results obtained by having one observer assess postures for extended periods of work. We believe that the budget constraints addressed in our study, i.e. cost allowances between $200 €$ and $600 €$ for 
collecting data from one individual, are realistic as a practitioner's scenario, e.g. in the occupational health service.

The results also show that the loss in information associated with choosing this one-observer strategy can be severe. Notably, our numeric results are sensitive to the sizes of unit costs and variance components, which are probably different for other jobs, sizes of the video recordings, observation procedures, and posture variables. The latter caveat is exemplified in our own study, since the loss in cost-efficiency associated with choosing a nonoptimal resource allocation was less severe for $\%$ time $>90$ than for $\%$ time $<15$.

We obtained our results using fairly simple models for assessing cost and statistical efficiency when assessing posture means of an individual. While the losses associated with not using an optimally cost-efficient measurement strategy may change with more elaborate models [18, 21], we have good reasons to claim that observer variability will remain an important determinant of how to appropriately allocate resources when addressing postures of individuals, and even so when estimating mean postures in groups. We thus believe from inspecting Eq. (2) and (3) that the finding of more observers being an attractive alternative to more video recordings may apply in general to posture observations, unless the costs associated with collecting video recordings are very small compared to the costs of observing them and, at the same time, the "biological" posture variability between days is large compared to the inter-observer variability. At present, it is not possible to judge under which circumstances this situation may occur, if ever.

Our results also suggest that posture assessment by observation should, preferably, be done on the basis of video recordings. The common practice of on-site observations does not permit repeated observations of the same work sequence, and thus precludes the opportunity to increase statistical precision by multiple observations. Since our study demonstrates that a one-observer one-view observation strategy can be highly inefficient, we strongly recommend work site visits for the purpose of assessing working postures to include recordings of the work on video for further analysis.

\section{Conclusion}

The present study demonstrated that when working postures are investigated by observation, it can be highly cost-efficient to spend resources on multiple repeated observations of a number of collected video recordings, rather than on collecting more videos and having them observed fewer times. This result should guide future observational posture data collections in research and practice into, (1) including recordings of the work on video, (2) engaging multiple observers to assess each recording.

\section{References}

[1] S. Bao, N. Howard, P. Spielholz, B. Silverstein and N. Polissar, Interrater reliability of posture observations, Hum Factors 51 (2009), 292-309.

[2] A. Burdorf and M. van Riel, Design of strategies to assess lumbar posture during work, Int J Ind Ergon 18 (1996), 239249.

[3] C.-C. Chen, C.-L. Chuang, K.-Y. Wu and C.-C. Chan, Sampling strategies for occupational exposure assessment under generalized linear model, Ann Occup Hyg 53 (2009), 509-521.

[4] D. Denis, M. Lortie and M. Rossignol, Observation procedures characterizing occupational physical activities: critical review, Int J Occup Safety Ergon 6 (2000), 463-491.

[5] N.B. Fethke, D. Anton, J.E. Cavanaugh, F. Gerr and T.M. Cook, Bootstrap exploration of the duration of surface electromyography sampling in relation to the precision of exposure estimation, Scand J Work Environ Health 33 (2007), 358-367.

[6] G.-Å. Hansson, I. Arvidsson, K. Ohlsson, C. Nordander, S.E. Mathiassen, S. Skerfving and I. Balogh, Precision of measurements of physical workload during standardised manual handling. Part II: Inclinometry of head, upper back, neck and upper arms, J Electromyogr Kinesiol 16 (2006), 125-136.

[7] M.J.M. Hoozemans, A. Burdorf, A.J. van der Beek, M.H.W. Frings-Dresen and S.E. Mathiassen, Group-based measurement strategies in exposure assessment explored by bootstrapping, Scand J Work Environ Health 27 (2001), 125-132.

[8] J.A. Jackson, S.E. Mathiassen and P.G. Dempsey, Methodological variance associated with normalization of occupational upper trapezius EMG using submaximal reference contractions, J Electromyogr Kinesiol 19 (2009), 416-427.

[9] K. Kazmierczak, S.E. Mathiassen, W.P. Neumann and J. Winkel, Observer reliability of industrial activity analysis based on video recordings, Int J Ind Ergon 36 (2006), 275282.

[10] G. Li and P. Buckle, Current techniques for assessing physical exposure to work-related musculoskeletal risks, with emphasis on posture-based methods, Ergonomics 42 (1999), 674-695. 
11] P. Liv, S.E. Mathiassen and S.W. Svendsen, Theoretical and empirical efficiency of sampling strategies for estimating upper arm elevation, Ann Occup Hyg 55 (2011), 436-449.

[12] S.E. Mathiassen and K. Bolin, Optimizing cost-efficiency in mean exposure assessment - cost functions reconsidered, BMC Med Res Methodol 11 (2011), 76.

[13] S.E. Mathiassen, A. Burdorf and A.J. van der Beek, Statistical power and measurement allocation in ergonomic intervention studies assessing upper trapezius EMG amplitude. A case study of assembly work, J Electromyogr Kinesiol 12 (2002), 27-39.

[14] S.E. Mathiassen, A. Burdorf, A.J. van der Beek and G.-Å. Hansson, Efficient one-day sampling of mechanical job exposure data - a study based on upper trapezius activity in cleaners and office workers, Am Ind Hyg Assoc J 64 (2003), 196-211.

[15] S.E. Mathiassen, T. Möller and M. Forsman, Variability in mechanical exposure within and between individuals performing a highly constrained industrial work task, Ergonomics 46 (2003), 800-824.

[16] S.E. Mathiassen and V. Paquet, The ability of limited exposure sampling to detect effects of interventions that reduce the occurrence of pronounced trunk inclination, Appl Ergon 41 (2010), 295-304.

[17] M. Rezagholi and S.E. Mathiassen, Cost-efficient design of occupational exposure assessment strategies - a review, Ann Occup Hyg 54 (2010), 858-868.
[18] M. Rezagholi, S.E. Mathiassen and P. Liv, Cost efficiency comparison of four video-based techniques for assessing upper arm postures, Ergonomics (submitted).

[19] S.J. Samuels, G.K. Lemasters and A. Carson, Statistical methods for describing occupational exposure measurements, Am Ind Hyg Assoc J 46 (1985), 427-433.

[20] E.P. Takala, I. Pehkonen, M. Forsman, G.-Å. Hansson, S.E. Mathiassen, W.P. Neumann, G. Sjøgaard, K.B. Veiersted, R.H. Westgaard and J. Winkel, Systematic evaluation of observational methods assessing biomechanical exposures at work, Scand J Work Environ Health 36 (2010), 3-24.

[21] C. Trask, S.E. Mathiassen, J. Wahlström, M. Heiden and M. Rezagholi, Modeling costs of exposure assessment methods in industrial environments, Present conference (2012).

[22] C. Trask, K. Teschke, J. Morrison, P.W. Johnson, J. Village and M. Koehoorn, How long is long enough? Evaluating sampling durations for low back EMG assessment, J Occup Environ Hyg 5 (2008), 664-670.

[23] J. Wahlström, S.E. Mathiassen, P. Liv, P. Hedlund, M. Forsman and C. Ahlgren, Upper arm postures and movements in female hairdressers across four full working days, Ann Occup Hyg 54 (2010), 584-594. 